\title{
Further isolations of non-flagellate Pseudomonas aeruginosa
}

\author{
F. W. LEE \\ From the Department of Pathology, Westwood Hospital, Beverley, Yorkshire
}

SYNOPSIS Three strains of non-flagellate Ps. aeruginosa isolated from pathological specimens have already been described. The purpose of this paper is to report further isolates. All strains have been shown to be different types isolated from a variety of infections.

Since the isolation of three non-flagellate strains of $P$ s. aeruginosa reported recently (Lee, 1973), I have during the course of routine microbiological investigations isolated further distinct strains from typical Pseudomonas infections. Despite exhaustive attempts to induce flagellation and motility, all six strains have remained unchanged. Other isolates were not motile on primary isolation but motility was induced by cultural methods.

\section{Isolations}

Non-flagellate Ps. aeruginosa was isolated from an infected ear, a urinary tract infection, and an operation wound, each isolate from a different patient. The strains are referred to as strains 4,5 , and 6 respectively.

\section{MATERIALS AND METHODS}

Primary isolation was made by inoculating the specimens directly onto nutrient blood agar and MacConkey agar and the organisms were identified using the biochemical and typing methods previously employed.

\section{INVESTIGATIONS FOR MOTILITY}

Since the original work on non-motile strains was initiated a standard procedure has been adopted. Twenty-four-hour peptone water cultures incubated at both 37 and $24^{\circ} \mathrm{C}$ were examined by the hanging-drop technique. Any motile isolates were discarded. Those showing no signs of motility were examined further. The centre tube of a bottle of Craigie's medium was inoculated using a straight wire and incubated at $37^{\circ} \mathrm{C}$ for four to six days. At the same time agar plates of motility test medium

Received for publication 9 April 1974.
EE (Difco) were seeded by multiple stab culture. These were examined after 18 and $36 \mathrm{hr}$ for hazy $\stackrel{\square}{\gtrless}$ zones around the stab line denoting motility.

Control motile strains were examined with all tests. The growth from Craigie tubes was examined $\overrightarrow{0}$ microscopically for motility; if it was not motile it was inoculated into motility test medium and a further Craigie tube.

\section{Results}

The strains 4,5 , and 6 were shown by biochemical tests, phage, pyocine, and serological typing methods to be Ps. aeruginosa. Electron microscopy was employed to study bacterial morphology. The three strains described here were non-flagellate whereas a control motile strain showed typical polar flagella. Other results are presented in tables I and II. During the examination of Ps. aeruginosa strains isolated from routine microbiology of hospital, general practice, and other sources of pathological speci-

\begin{tabular}{llll}
\hline $\begin{array}{l}\text { Cultural and Biochemical } \\
\text { Characteristics }\end{array}$ & \multicolumn{2}{l}{ Strain Number } \\
\cline { 2 - 3 } & 4 & 5 & 6
\end{tabular}

Anaerobic growth

Green pigment on nutrient agar

Trimethylamine odour

Growth at $42^{\circ} \mathrm{C}$

Growth on cetrimide agar

Oxidase (Kovacs)

Catalase

Nitrate reduction to gaseous nitrogen

Arginine dihydrolase

Gluconate oxidation

Gelatin liquefaction

Colony

\begin{tabular}{|c|c|c|}
\hline- & - & - \\
\hline+ & + & + \\
\hline+ & + & + \\
\hline+ & + & + \\
\hline+ & + & + \\
\hline+ & + & + \\
\hline+ & + & + \\
\hline+ & + & + \\
\hline+ & + & + \\
\hline- & - & - \\
\hline $\begin{array}{l}+ \\
\text { Rough }\end{array}$ & $\stackrel{+}{\text { Mucoid }}$ & $\begin{array}{l}+ \\
\text { Rough }\end{array}$ \\
\hline
\end{tabular}

Table I Cultural and biochemical properties of the non-flagellate Ps. aeruginosa strains 


\begin{tabular}{llll}
\hline $\begin{array}{l}\text { Typing } \\
\text { Method }\end{array}$ & \multicolumn{2}{l}{ Strain Number } & \\
\cline { 2 - 4 } & 4 & 5 & 6 \\
\hline Pyocine & 3 & 1d & 3 \\
Phage & Lysis + & No lysis & Lysis + \\
Serological & 6 & Not typable & 7 \\
\hline
\end{tabular}

Table II Results of typing methods

mens, most were motile after incubation in peptone water at $37^{\circ} \mathrm{C}$. Occasional strains were isolated, however, that were not motile or only sluggishly motile on primary isolation. Active motility was induced by passage through Craigie's medium. Motility test medium EE (Difco) was found to be invaluable in these tests, a motile strain having a large hazy growth around the stab inoculum, a non-motile strain no such growth. Some of the feebly motile strains produced very small zones but these were readily seen when incubation was repeated. All the feebly motile forms were shown to be actively motile after passage through Craigie tubes. Despite repeated passage through Craigie tubes and repeated examinations by hanging drop methods and multiple stab inocula into motility medium, the phenomenon of motility was not induced by strains 4,5 , and 6 .

\section{Discussion}

Six distinct strains of non-flagellate Ps. aeruginosa have been isolated as prime pathogens as a result of routine work in a small hospital over a period of one year. The fact that the strains are different and that they are being isolated from more than one source and from a variety of infections raises the question of how common non-flagellate $P$ s. aeruginosa is in pathological sites. Approximately 60 actively motile strains were also isolated during this study. Many laboratories do not examine all isolates of Pseudomonas for motility and flagellation. It seems to me that the categorical definition of Ps. aeruginosa as motile rods is suspect, especially if these findings are corroborated elsewhere.

I should like to thank Dr M. T. Parker for phage and serological typing, Mrs J. E. Mundy for electron microscopy, and Mrs V. M. Addey and Mr E. Boyd for their technical help.

Reference

Lee, F. W. (1973). Non-flagellate Pseudomonas aeruginosa in pathological material. J. clin. Path., 26, 826-827. 